\title{
Spinal cord compression due to intrathoracic extramedullary haematopoiesis in homozygous thalassaemia
}

\author{
AW IBRAHIM, EM IBRAHIM, NM MITRY, A ABDUL SATIR, A KUPPA \\ From the King Faisal University, College of Medicine and Medical Sciences, Dammam, Saudi Arabia
}

SUMMARY A rare condition of massive intrathoracic extramedullary haematopoiesis causing spinal cord compression is presented in a 17-year-old Saudi male. Both clinical and laboratory findings, together with the computed tomographic evaluation of the lesion are described. Partial surgical resection as an alternative to radiotherapy has resulted in a dramatic response.

Extramedullary haematopoiesis is a compensatory phenomenon that occurs when the normal function of the bone marrow is disturbed. The organs that are most frequently involved include the spleen, liver, and lymph nodes. However, other organs may also be involved but less frequently. The thorax is not a common site for extramedullary haematopoiesis and in the English literature only 55 cases of intrathoracic extramedullary haematopoiesis have been reported. ${ }^{12}$ The age range of affected patients was 8-81 yr with a mean of 43 . Thalassaemia was the commonest cause in all those reported cases. Among those patients with intrathoracic extramedullary haematopoiesis, only three presented with spinal cord compression due to epidural haematopoiesis. ${ }^{34} \mathrm{We}$ present here a case of thalassaemia intermedia presenting with advanced paraparesis that responded well to surgical treatment. Although, it has been shown that extramedullary haematopoiesis tissue is highly sensitive to small doses of radiation, ${ }^{4}$ surgery in the absence of this facility can produce an equally good result.

\section{Case report}

A 17-year-old Saudi Arabian male farmer presented with a one year history of mid-dorsal backache. The pain was

Address for reprint requests: Dr EM Ibrahim, King Faisal University, College of Medicine and Medical Sciences, PO Box 2114, Dammam, Saudi Arabia.

Received 19 November 1982 and in revised form 12 February 1983.

Accepted 19 March 1983 dull aching in nature made worse by movement, exertion and cough and relieved by rest. The pain did not radiate to the chest wall or to the lower limbs.

He described the pain as mild at first but later it became more severe. Four weeks prior to his hospital admission, he complained of progressive weakness of both lower limbs and finally was unable to walk or support his weight. There was no urinary or bowel disturbance. The patient had no significant past medical history, and no family history of a similar complaint was elicited.

Physical examination revealed a thin, pale and ill looking young man who was less physically developed than his stated age. In the upper limbs the humeri looked short in relation to his forearms. The chest wall showed an obvious pigeon-shaped deformity and swelling of all costochondral junctions. The spleen was palpable two fingers breath below the costal margin but the liver was not enlarged. The positive findings on CNS examination were confined to his lower limbs. He had a spastic paraparesis with grade 4 diffuse and bilateral muscle weakness. Hyperreflexia and extensor plantar responses with a sensory level corresponding to D7 were elicited. Laboratory investigations gave the following results: haemoglobin $10 \mathrm{gm} / \mathrm{dl}$, HCT $36 \%$, MCV $62 / \mathrm{m} \mathrm{m}^{3}, \mathrm{MCH} 20 / \mathrm{pg}, \mathrm{MCHC} 30 \%$, and normal WBC and differential. The blood film showed hypochromia, microcytosis, polychromasia, target cells, poikilocytosis, anisocytosis, and nucleated erthrocytes. Sickle test was negative. Serum iron was normal. His chest radiograph (fig 1a) showed a paraspinal well demarcated mass at the level of T4 on the right side and a similar mass at T10 level. Myelogram demonstrated a total block at T7/T8 due to an extradural posteriorly placed mass. The extent of the mass was confirmed by a CT scan, (fig 1b); it was found to extend from T4 to T8. Cellulose acetate haemoglobin electrophoresis in alkaline medium demonstrated haemoglobin F and haemoglobin A2 without haemoglobin A. By the Sniger's alkali denaturation, haemoglobin $\mathrm{F}$ was $72 \%$. 


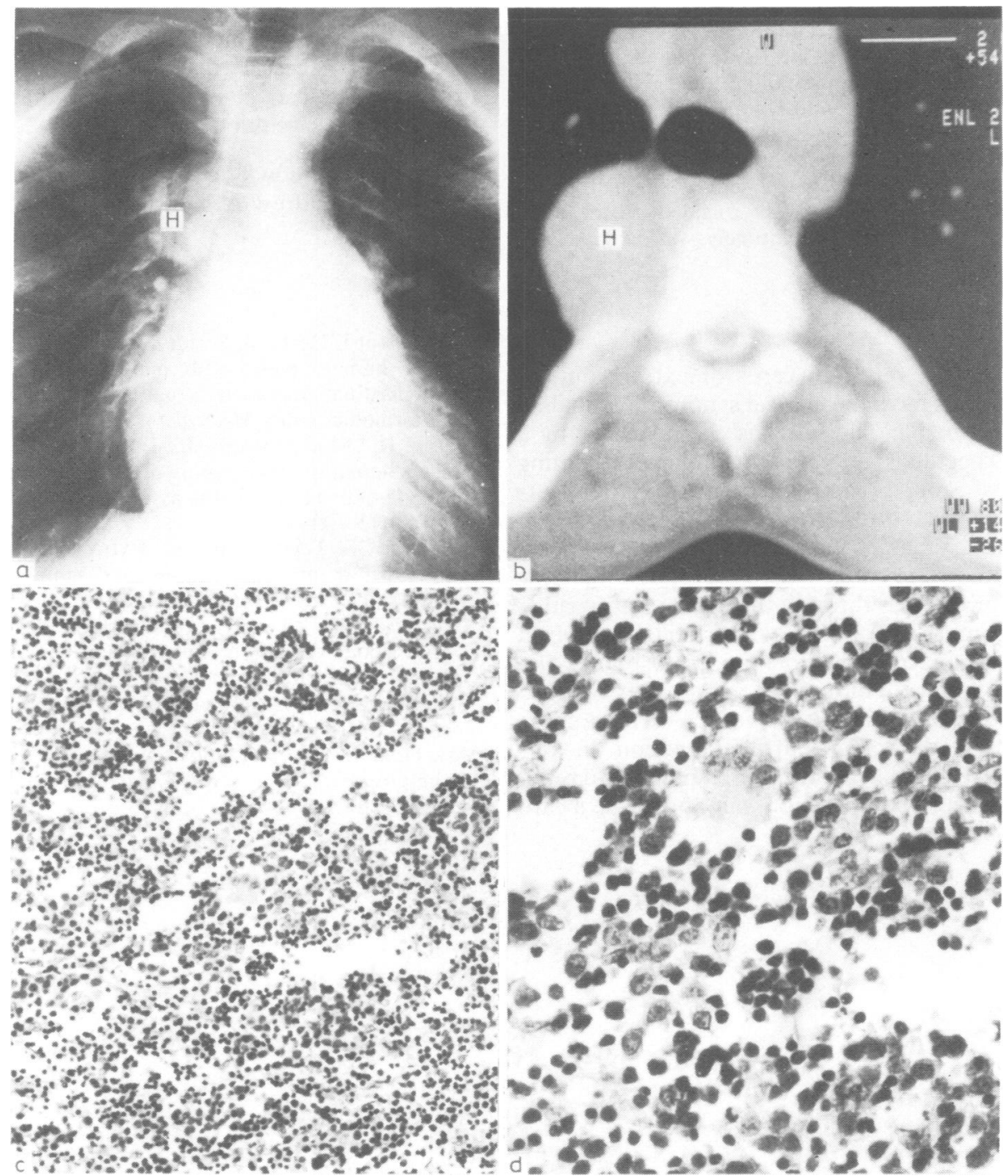

Fig 1 (a) PA chest radiograph demonstrating the intrathoracic extramedullary haematopoietic tissue $(H)$. (b) CT scan of the paravertebral extramedullary haematopoiesis tissue $(H)$. In the spinal canal, another mass is seen displacing the cord anteriorly and it seems to be continuous with the paravertebral mass. (c) Megakaryocyte surrounded by histiocytes and mononuclear cells $(H \& E \times 50)$. (d) Higher magnifiction of (c) showing megakaryocytes surrounded by macrophages, lymphocytes, plasma cells, and leucocytes $(H \& E \times 200)$.

Haemoglobin A2 by elution and spectrophotometry after cellulose acetate electrophoresis was $5 \cdot 2 \%$. Densitometry of haemoglobin electrophoresis revealed a haemoglobin $\mathrm{F}$ of $92 \%$. The discrepancy between standard alkali denaturation test and electrophoresis of haemoglobin $F$ is well known. It was safe to assume that haemoglobin $\mathbf{A}$ was absent in this patient. Haemoglobin distribution (Kleihauer-Betke technic), was heterogeneous. A diagnosis of homozygous $\mathbf{B}^{\circ}$-thalassaemia was established.
In view of the laboratory and radiological findings, the spinal cord compression was believed to be due to intrathoracic extramedullary haematopoiesis tissue as a complication of thalassaemia. Due to lack of radiotherapy facilities at the present time, surgery was the only alternative. During exploration the laminae were found to be soft and vascular. A bluish soft and vascular extramedullary mass was found displacing the spinal cord anteriorly. Most of the mass causing the maximum compression was 
removed and it was felt that bleeding would hinder any attempt at a more extensive excision.

Microscopy of the multiple soft tissue fragments showed haematopoietic tissue composed of both red cell and white cell precursors, and mixed with lymphocytes, plasma cells and histiocytes. Eosinophils and megakaryocytes were prominent (fig 1c, d). There was no evidence of neoplasm. Four weeks postoperatively the patient was free from all symptoms and was able to walk unaided. After nine months the patient is doing extremely well and has no recurrence of his symptoms.

\section{Discussion}

The majority of reported cases of extramedullary haematopoiesis have the clinical syndrome of thalassaemia intermedia. The latter is characterised by a long life expectancy, and mild anaemia necessitating blood transfusion very infrequently. Our patient certainly falls in this category. The origin of extramedullary haematopoiesis is obscure. A possible explanation is that the hyperplastic marrow herniates or extends through the thinned trabeculae in the proximal ends of the ribs or from the vertebral bodies. ${ }^{5}$ An alternative explanation postulates the transformation of embryonic rests of tissue into haemopoietic tissue in circumstances of bone marrow stress, ${ }^{26}$ Although partial resection in our patient has resulted in a dramatic response and relief of the spinal cord compression, one should be aware of the clinical situation together with the typical radiological findings that allow correct diagnosis and thus avoid unnecessary diagnostic needle aspirate or surgery which can cause dangerous haemorrhage from a highly vascular mass.

The authors acknowledge the expert technical assistance of Mr Andrew Archibald and Mrs Nilda Tavera.

\section{References}

${ }^{1}$ Ben-Bassat I, Hertz M, Selzer G, Ramot B. Extramedullary hematopoiesis with multiple tumor-simulating mediastinal masses in a patient with B-thalassemia intermedia. Israel Medical J 1977;13:1206-10.

${ }^{2}$ Verani R, Oslon J, Moake JL. Intrathoracic extramedullary hematopoiesis: report of a case in a patient with sickle-cell disease B-thalassemia. Am J Clin Pathol 1980;73:133-7.

${ }^{3}$ Sorsdahl OS, Taylor PE, et al. Extramedullary hematopoiesis, mediastinal masses and spinal cord compression. JAMA 1964;189:343.

${ }^{4}$ Luyendijk W, Went L, Schadd H. Spinal cord compressional due to extramedullary hematopoiesis in homozygous thalassemia. J Neurosurg 1975;42:212.

${ }^{5}$ Case Records of the Massachusetts General Hospital. $N$ Engl J Med 1963;278:782.

${ }^{6}$ Ask-Upmark E. Tumour simulating intrathoracic heterotopia of bone marrow. Acta Radiol 1945;26:425-440. 